\title{
Detecting beer intake by unique metabolite patterns
}

Gürdeniz, Gözde; Jensen, Morten Georg; Meier, Sebastian; Bech, Lene; Lund, Erik; Dragsted, Lars Ove

Published in:

Journal of Proteome Research

Link to article, DOI:

10.1021/acs.jproteome.6b00635

Publication date:

2016

Document Version

Peer reviewed version

Link back to DTU Orbit

Citation (APA):

Gürdeniz, G., Jensen, M. G., Meier, S., Bech, L., Lund, E., \& Dragsted, L. O. (2016). Detecting beer intake by unique metabolite patterns. Journal of Proteome Research, 15(12), 4544-4556.

https://doi.org/10.1021/acs.jproteome.6b00635

\section{General rights}

Copyright and moral rights for the publications made accessible in the public portal are retained by the authors and/or other copyright owners and it is a condition of accessing publications that users recognise and abide by the legal requirements associated with these rights.

- Users may download and print one copy of any publication from the public portal for the purpose of private study or research.

- You may not further distribute the material or use it for any profit-making activity or commercial gain

- You may freely distribute the URL identifying the publication in the public portal

If you believe that this document breaches copyright please contact us providing details, and we will remove access to the work immediately and investigate your claim 


\section{Detecting beer intake by unique metabolite patterns}

Gözde Gürdeniz ${ }^{*}$, Morten Georg Jensen², Sebastian Meier ${ }^{3}$, Lene Bech², Erik Lund ${ }^{2}$, and Lars Ove Dragsted $^{1}$

${ }^{1}$ Department of Nutrition, Sports and Exercise, Faculty of Science, University of Copenhagen, Denmark. ${ }^{2}$ Carlsberg Research Laboratory A/S, Gamle Carlsberg Vej 6-10, 1799 Copenhagen V, Denmark, ${ }^{3}$ Department of Chemistry, Technical University of Denmark, Kemitorvet, 2800 Kgs. Lyngby, Denmark

*Corresponding author:

Gözde Gürdeniz

Address : Rolighedsvej 30, Frederiksberg C, 1958, DENMARK

e-mail : gozg@nexs.ku.dk

phone : +4535332372 


\section{Abbreviations}

AA : Alpha acids

ABV : Alcohol By Volume

ASCA : ANOVA simultaneous component analysis

$\mathrm{C}:$ Control beverage (soft drink)

IAA : Iso- $\alpha$-acids

IBU : International Bitterness Units

LB : Light beer

MSt1 : Meal Study 1

MSt2 : Meal Study 2

NMR : Nuclear magnetic resonance spectroscopy

NMT : N-methyl tyramine

pGlu-Pro : Pyro-glutamyl proline

PLSDA: Partial least squares discriminant analysis

RB : Regular beer

SCA : Simultaneous component analysis

SS : Sum of squares

UPLC-MS : Ultra-performance liquid chromatography quadrupole time-of-flight mass spectrometry 


\begin{abstract}
Evaluation of health related effects of beer intake is hampered by the lack of accurate tools for assessing intakes (biomarkers). Therefore, we identified plasma and urine metabolites associated with recent beer intake by untargeted metabolomics and established a characteristic metabolite pattern representing raw materials and beer production as a qualitative biomarker of beer intake. In a randomized, crossover, single-blinded meal study (MSt1) 18 participants were given one at a time four different test beverages: strong, regular and non-alcoholic beers and a soft drink. Four participants were assigned to have two additional beers (MSt2). In addition to plasma and urine samples, test beverages, wort and hops extract were analyzed by UPLC-QTOF. A unique metabolite pattern reflecting beer metabolome, including metabolites derived from beer raw material (i.e. N-methyl tyramine sulfate and the sum of iso- $\alpha$-acids and tricyclohumols) and production process (i.e. pyro-glutamyl proline and 2ethyl malate) were selected to establish a compliance biomarker model for detection of beer intake based on MSt1. The model predicted the MSt2 samples collected before and up to $12 \mathrm{~h}$ after beer intake correctly $(\mathrm{AUC}=1)$. A biomarker model including four metabolites representing both beer raw materials and production steps provided a specific and accurate tool for measurement of beer consumption.
\end{abstract}

Keywords: Beer, Barley, Hops, Biomarker model, Hops, Metabolomics, Plasma, Urine, UPLC-QTOF 


\section{Introduction}

Light to moderate alcohol consumption has been associated with decreased total mortality and with lower risk of metabolic diseases such as diabetes and cardiovascular disease ${ }^{1,2}$, but also with adverse effects on certain cancers ${ }^{3}$. The health related effects of consuming alcoholic beverages are mostly attributed to their alcohol content rather than specific components of different kinds of beverages, although there are some contradictory findings ${ }^{4,5}$. One of the major issues in alcohol research is the assessment in observational studies of the consumption of specific alcoholic beverages usually obtained using self-reported questionnaires. These tools may provide only moderately reliable information, since reporting may be influenced by cultural and personal views on alcohol. Biomarkers of food intake are powerful tools for assessment of dietary exposure in clinical and observational studies. Some recent studies suggested resveratrol and isoxanthohumol as intake biomarkers for wine and beer consumption, respectively $^{6,7}$. However, resveratrol is also found in grapes and berries and isoxanthohumol has a highly variable rate of biotransformation into 8-prenylnaringenin $(0-100 \%$ conversion) in the human gut making it a less than ideal biomarker ${ }^{8,9}$.

Beer is one of the most widely consumed beverages and is a very complex food with a metabolome originating from raw materials (e.g. barley and hops) and their transformation products formed during malting, boiling and fermentation. Consumption of beer may lead to direct absorption of its components but quite often their degradation or biotransformation products have also to be taken into consideration. Hops may be the most characteristic ingredient in beer and therefore the best candidate for single biomarker search strategies ${ }^{10}$. Iso- $\alpha$-acids (IAAs) from hops, are specific to beer ${ }^{11,12}$ yet one major issue with IAAs as biomarkers is their high susceptibility to degradation, leading to multiple products; therefore their quantity varies after storage ${ }^{13,14}$. In terms of metabolism of IAAs, only limited 
number of studies is available ${ }^{15,16}$. Therefore investigating the fate of not only IAAs but also their oxidation products in the human body would be a prerequisite for finding a suitable hops-derived biomarker.

Basic steps and raw materials in beer production are mostly universal, yet production processes by different producers vary, thereby further complicating the search for a global beer biomarker. Furthermore, human individuals differ with respect to many biotransformation pathways, making it even more challenging to find any single biomarker that is universal for beer intake. Finding markers representing each common raw material and step in beer production and mapping their biotransformation may therefore be a strategy to design more global aggregated biomarkers, representing the complete process. Beer as well as alcohol may affect metabolism by altering the glucose and insulin responses ${ }^{17},{ }^{18}$ and these parameters are therefore of potential importance as modifiers of biomarker responses.

Metabolomics resulting from the development of highly sensitive modern analytical instruments is an attractive tool for identification of novel intake biomarkers for specific diets, foods, food constituents and/or for further assessment of their health impacts ${ }^{19-22}$. This technology together with chemometrics also allows the identification of metabolite patterns as markers of intake ${ }^{19}$. Therefore, utilizing an LCMS based metabolomics and chemometrics approach we aimed here to identify the plasma and urinary metabolites associated with recent beer intake and establish a novel and validated aggregated biomarker produced by combination of identified metabolites that represent each of the raw materials, processing steps, thereby ascertaining uniqueness to beer intake. 


\section{Materials and Methods}

\section{Subjects}

37 healthy subjects within an age range of 18-60 years were recruited from the greater Copenhagen area by bulletins at educational institutions and on public websites for recruiting volunteers for clinical studies. Exclusion criteria were the following: systemic infections, psychiatric conditions and metabolic disease; alcohol intolerance; alcohol intake above the Danish Health and Medicines Authority's recommended limits of 7 and 14 units per week for females and males, respectively; donation of blood (>24 ml) less than 3 months before the study; breast feeding or pregnancy.

The subjects were given both verbal and written information about the study before providing their written consent. The study was carried out at the Department of Nutrition, Exercise and Sports, Faculty of Science, University of Copenhagen, Frederiksberg, Denmark. The research protocol was approved by the Ethical Committee of the municipalities of Copenhagen and Frederiksberg (H-1-2013-029) and the study has been registered at clinicaltrials.gov (NCT02449577).

\section{Study Designs and Diets}

Meal study 1(MSt1): 19 subjects were included in the main part of the study. A formal power calculation is not possible for explorative studies but several previous meal studies have indicated that 10-20 volunteers suffice. The study was performed as a randomized, crossover, single-blinded intervention with four study periods of three days and a wash-out period of $>1$ week between periods. The four periods consisted of a test day where subjects were served with one of the three different test beers or a control beverage in randomized order. The three test beers were a strong lager (SB) (Carlsberg Elephant, 8 \% ABV, 35 IBU), a regular lager (RB) (Carlsberg Pilsner, $4.6 \%$ ABV, 22 
IBU), and a light/alcohol-free beer (LB) (Tuborg Super Light $0.1 \%$ ABV, 19 IBU). The control soft drink beverage (C) (Carlsberg Sports drink) was alcohol free but with the same energy content (carbohydrate and protein) as the average of the test beers.

The evening before the test day, subjects consumed a standardized dinner (spaghetti Bolognese) with water ad libitum. On the test day, after having a standardized breakfast (bread, butter, ham, cheese, orange juice) ad libitum between 7-8 am, subjects were instructed to fast for 3-4 hours (except for $\sim 1 / 2$ liter of water). Subjects were served a standardized lunch of Parisian toast/grilled cheese sandwiches (Tulip Food Company) at 11.35 am together with one of the test beers or the $\mathrm{C}(330 \mathrm{ml})$, all to be consumed within $25 \mathrm{~min}$. The amount of toast was adjusted according to the type of drink served, so that the content of macronutrients was the same in each meal. Until $2.35 \mathrm{pm}$ (last blood sampling) subjects did not consume any food or drink, but had water ad libitum. Thereafter, subjects were allowed to consume their habitual diet but were instructed to abstain from intake of alcohol in any form for 24 hours after the start of each test day (i.e. until 8 am in the following morning). They were otherwise instructed to retain their usual pattern of alcohol intake during the remaining 4 weekdays between each of the four test periods.

Meal study 2(MSt2): Four subjects were included in a second (validation) study; one of them also participated in the MSt1. The study was performed as a randomized, crossover intervention with all the same details as for MSt1, except that only two study periods of three days were conducted. The two periods consisted of a test day where subjects were served with two servings $(660 \mathrm{ml})$ of either of the two test beers in randomized order. Two test beers were used; one being a high hops moderate alcohol beer (HHB) (Lawn Mower, Carlsberg breweries, Sweden, $4.8 \% \mathrm{ABV}, \sim 31 \mathrm{IBU}$ ) and the other a low hops high alcohol beer (LHB) (T-beer, Tuborg breweries, Romania7-8 \% ABV, 8 IBU). These beers 
were selected as extremes with regard to the two main raw materials in beer to serve as a test for the validity of the biomarker development concept.

On the test day volunteers in the MSt2 validation study had a standardized breakfast and lunch (multigrain bread containing also barley, cheese, ham, a red pepper fruit, radishes, apple, banana, orange juice and egg) according to calculated energy needs, and consumed the same standardized dinner together with $660 \mathrm{ml}$ of either of the test beers. The following day, they consumed the same standardized meals but not the test beers.

In both studies volunteers were allowed otherwise to remain on their habitual diets, but were required to abstain from drinking all forms of alcohol and avoid heavy physical activity from 48 and 24 hours before the test days, respectively.

A simple randomization was used in both studies. The brewery delivered the four drinks in dark bottles, marked A, B, C, D for MSt1 and E and F for MSt2. A sequence table was generated for all volunteers so that they received the drinks in random order within each study. In principle participants, care providers and assessors were all blinded, however the participants could easily taste the nature of the soft drink. They were instructed not to tell the care providers.

\section{Blood and Urine Sampling}

Blood samples were drawn only in MSt1 on each test day $20 \mathrm{~min}$ before (baseline) and $0.45,2$ and $3 \mathrm{~h}$ after the subjects consumed the test beverages together with a standardized lunch. The blood samples were transferred into $4 \mathrm{~mL}$ vials containing heparin as an anticoagulant. The blood was centrifuged at $3,000 \mathrm{~g}, 4^{\circ} \mathrm{C}$ for $10 \mathrm{~min}$. The plasma fraction was aliquoted into $2 \mathrm{~mL}$ cryotubes and stored at $-80^{\circ} \mathrm{C}$ until further processing. 
Urine samples were collected on each test day within the following time periods: from their morning void until $20 \mathrm{~min}$ before having their test drink (baseline); from baseline until $1.5 \mathrm{~h}$; from $1.5 \mathrm{~h}$ to $3 \mathrm{~h}$; and from $3 \mathrm{~h}$ to $21 \mathrm{~h}$. In addition, $24 \mathrm{~h}$ pooled samples were created as a mixture of the urine samples collected in the four different time intervals in relative proportion to their volumes.

For the MSt2, only urine samples were collected: from lunch to dinner before having the test beer (baseline), baseline to $2 \mathrm{~h}, 2 \mathrm{~h}$ to $4 \mathrm{~h}, 4 \mathrm{~h}$ to $12 \mathrm{~h}, 12 \mathrm{~h}$ to $24 \mathrm{~h}$ and $24 \mathrm{~h}$ to $40 \mathrm{~h}$.

During sampling, urine was stored below $5^{\circ} \mathrm{C}$ in cooling bags and after completion, sample aliquots were kept at $-80{ }^{\circ} \mathrm{C}$ until further analysis.

\section{Blood glucose and serum insulin}

The blood samples were collected in dry tubes for the measurement of insulin and in fluoride citrate tubes for the measurement of glucose. Plasma glucose was measured with an enzymatic colorimetric method (ABX Pentra Glucose HK CP; Horiba, Montpellier, France) and analyzed with an ABX Pentra 400 (Horiba). Serum insulin was measured by a chemiluminescent immunometric assay with an Immulite 1000 Immunoassay System (Siemens Healthcare Diagnostics, West Sacramento, CA). All samples were analysed in one batch. Intra-assay and interassay coefficients of variation (CVs) for glucose and insulin were below $2.8 \%$.

\section{UPLC-QTOF Analysis}

Plasma protein precipitation, urine centrifugation and dilution procedures were performed as described previously ${ }^{23,24}$. The 288 plasma samples and 320 urine samples were placed in 96-well plates, such that all samples (urine or plasma) from the same individual were in the same plate (analytical batch) 
while the individuals were randomized between batches (five per batch) and the order of samples was randomized within each batch. An ultra-performance liquid chromatography (UPLC) system coupled to a quadrupole time-of-flight mass spectrometer (Premier QTOF, Waters Corporation, Manchester, UK) was used for sample analysis. The mobile phase was $0.1 \%$ formic acid in water (solvent A) and $0.1 \%$ formic acid in $70 \%$ acetonitrile and $30 \%$ methanol (solvent B). Five $\mu \mathrm{L}$ of each sample were injected into a HSS T3 C18 column $(2.1 \times 100 \mathrm{~mm}, 1.8 \mu \mathrm{m})$ coupled with a VanGuard HSS T3 C18 column (2.1 $\mathrm{x} 5 \mathrm{~mm}, 1.8 \mu \mathrm{m})$ and the gradient was operated for $7.0 \mathrm{~min}$. The eluate was analyzed by electrospray ionization (ESI) in positive and negative mode, applying a capillary voltage of $3.2 \mathrm{kV}$ and $2.8 \mathrm{kV}$, respectively and cone voltage of $20 \mathrm{kV}$. Ion source and desolvation gas (nitrogen) temperatures were set to 120 and $400^{\circ} \mathrm{C}$, respectively. More detail on UPLC-QTOF analysis conditions can be found elsewhere $^{25}$. Blanks (5\% of acetonitrile:methanol $70: 30 \mathrm{v} / \mathrm{v}$ in water) and external metabolomics standard mixtures were injected after every 30 samples, throughout each analytical batch ${ }^{25}$.

In addition to plasma and urine samples, the test drinks, a beer wort extract, and hops extracts were analyzed with UPLC-QTOF using identical conditions. Sample preparation was comprised of dilution in solvent $\mathrm{A}$ for test drinks and wort whereas a mixture of solvent $\mathrm{A}$ and $\mathrm{B}(50-50 \%)$ was used for dilution of the hops extract.

MS/MS fragmentation experiments were conducted for structural characterization of beer intake markers. The collision-induced dissociation (CID) was set to 10, 20 and $30 \mathrm{eV}$ in separate runs and all other parameters were kept as for the MS full scan experiments as described above.

\section{Data Preprocessing}


Initially, raw data were converted to intermediate MZdata using an $\mathrm{R}$ function (convert.waters.Rd, https://github.com) operating on $\mathrm{R}$ software (ver. 3.1.0.). MZdata files were imported into MZmine 2.10 for data preprocessing including the following steps: mass detection, chromatogram builder, chromatogram deconvolution (local minimum search), isotopic peaks grouper, peak alignment (join aligner), duplicate peak filter, peak list row filter and gap filling (peak finder). The term 'feature' is used to refer to an ion with a specific retention time and mass over charge ratio $(\mathrm{m} / \mathrm{z})$ throughout this paper.

MZmine-preprocessed data were imported into MATLAB R2013a (ver. 8.1.0.604). Feature filtering was applied based on three criteria. First, the features detected earlier than 0.3 min and later than 6 min were removed. Second, if a feature has a significant peak area ( $>60$, approx. 6 times the maximal background noise) in the first blank sample in at least one of the analytical batches, the feature was removed from the entire set as background noise. Third, if a feature had a peak area lower than 10 (considered as noise level or gap filling errors), in more than $60 \%$ of the samples within every sample group (specified with one test drink type and one time point), the feature was excluded (percent rule).

Urine samples were normalized to unit-length normalization to correct for urine concentration variations. Later, to remove inter-batch variation, each feature detected in urine/plasma samples within each batch was divided with the overall mean of its recordings included in the entire batch. This approach is justified by randomization of individuals between batches and of all samples from each individual within the same batch prior to analysis. Application of these pre-processing and normalization procedures separately on negative and positive mode data was followed by concatenation of the datasets from the two modes. In order to ease identification, features within the retention time 
range of 0.02 were grouped and assigned as one feature group if they had correlation coefficients higher than 0.7 . Then, each feature group is considered as representation of one unique compound.

\title{
Data Analysis
}

\begin{abstract}
ASCA
Data analysis was performed in MATLAB R2013a (ver. 8.1.0.604). For the plasma samples the baseline level of each feature was subtracted from its subsequent recordings at each time point $(0.45,2$ and $3 \mathrm{~h}$ ) in order to reduce the influence of intra-individual factors. For urine, this procedure did not affect the outcome and was therefore omitted (data not presented).
\end{abstract}

Unit scaled data were subjected to, ASCA (ANOVA Simultaneous Component Analysis) ${ }^{26}$, to isolate and further investigate the variation related to test drinks and to identify the contributions of time course and test drink $\times$ time interactions.

In order to evaluate the significance of ASCA decomposed effect matrices, a permutation test was employed $^{27}$. The permutation test is based on comparison of sum of squares (SS) of each effect matrix with SS distribution of matrices obtained with repeated random permutation of class labels. In this case, the number of permutations was 100,000. The significance of each effect matrix was determined as the fraction of data permuted matrices that had SS higher than the SS of the original effect matrix.

Later, simultaneous component analysis (SCA) was applied on individual effect matrices to interpret the associated variation. SCA provides information only on the level averages, and to analyze the variation between samples within each level, the residuals were projected on the principal component subspace of effect matrices, as described previously ${ }^{28}$. 
An approach similar to ANOVA-PCA was carried out, where the residual matrix was added to test drink effect matrix ${ }^{20,28}$. This provided the basis data matrix for the subsequent PLSDA.

ASCA was performed with in-house developed MATLAB functions.

\section{PLSDA}

PLS_Toolbox (version 6.5, Eigenvector Research, Inc., MA, US) was used for partial least squares discriminant analysis, PLSDA. PLSDA was applied to differentiate the plasma and urine metabolic patterns associated with beer intake (i.e. $\mathrm{SB}+\mathrm{PB}+\mathrm{LB}$ vs. C) within the consumed beers. In order to validate PLSDA models, ten different test sets each comprised of data from all samples (time points and treatments) from all possible combinations of two subjects were set aside. Variable selection was performed repeatedly by removing variables with selectivity ratio and variable importance in projection (VIP) values lower than 1 until no further increase in the cross-validation (eight-fold) classification errors could be observed. Final models with selected variables were evaluated using test set misclassification $^{29}$. The variables that were selected in at least seven of the models were assigned as markers of beer intake.

\section{Calibration and validation models}

The calibration set was created from MSt1 including only the measurements of the selected metabolites for urine collected at 0-1.5 h, 1.5-3 h and 3-21 h. PLSDA was applied on the calibration set to discriminate the three beers $(\mathrm{SB}+\mathrm{RB}+\mathrm{LB})$ from the soft drink, $\mathrm{C}$. PLSDA models were built with 1 to 5 LV and optimum model complexity (i.e. number of components) was decided by evaluating misclassifications. This PLSDA model is used as the calibration model. MSt2 with the measurements 
of selected metabolites for four subjects was used as the test set to validate the calibration model. The evaluation was based on how well the calibration model performs in classifying MSt2 samples collected after beer intake within the beer periods (i.e. $\mathrm{SB}+\mathrm{RB}+\mathrm{LB}$ in MSt1), as well as classifying MSt2 samples collected before beer intake within C (MSt1). Models were assessed by diagnostic statistics, area under receiver operating characteristic curve (AUC) and misclassification rate.

\section{Standards and Identification}

Four levels of chemical identification according to the published guidelines for chemical analysis were used $^{30}$. Amino acids (i.e., tyrosine, leucine/isoleucine, proline) and the dipeptide pyroglutamyl proline (pGlu-Pro) were identified using an in-house metabolite database containing retention time information and MS spectra of reference substances. Reference standards for ICS-I3 (containing $62.3 \% \mathrm{w} / \mathrm{w}$ of trans-IAA) and ICE-3 (containing $44.64 \%$ w/w of AA) were obtained from Labor Veritas (Zurich, Switzerland). Isoxanthohumol and naringenin were purchased from Sigma (Schnelldorff, Germany). For unknown compounds with a signal area higher than 600, MS/MS spectra $(10,20$ and $30 \mathrm{eV})$ were recorded in order to obtain fragmentation information to compare with data in available databases, such as Metlin (https://metlin.scripps.edu), hmdb (http://www.hmdb.ca), Chembank (http://chembank.broadinstitute.org/welcome.htm), Chemspider (http://www.chemspider.com), and similar sites. All six test drinks and beer wort and hop extract were kindly provided by Carlsberg A/S.

\section{NMR analysis}

The presence and identity of the dipeptide pGlu-Pro in beer was verified by 2D homo- and heteronuclear high-resolution NMR spectroscopy using beer fractionated by preparative UPLC. To this end, lyophilized UPLC fractions containing compound with a mass consistent with possible pGlu-Pro 
were redissolved in $500 \mu \mathrm{L} \mathrm{D} \mathrm{D}_{2} \mathrm{O}$ (Cambridge Isotope Laboratories, Andover, MA, USA). NMR spectra including ${ }^{1} \mathrm{H}_{-}{ }^{13} \mathrm{C}$ HSQC and ${ }^{1} \mathrm{H}-{ }^{1} \mathrm{H}$ TOCSY were recorded on a Bruker Avance II $800 \mathrm{MHz}$ spectrometer equipped with a TCI Z-gradient CryoProbe and an $18.7 \mathrm{~T}$ magnet (Oxford Magnet Technology, Oxford, U.K.). All NMR spectra were recorded, processed and analysed with Bruker Topspin 2.1 (Fällanden, Switzerland).

\section{Selection of the aggregated biomarker for beer intake}

Initially, the origin of each compound associated with beer intake was grouped based on their origin. This is accomplished by comparing $\mathrm{m} / \mathrm{z}$ and retention time of each beer marker with $\mathrm{m} / \mathrm{z}$ and retention time of the compounds detected in test beers, hops extract and wort reference samples. Accordingly, beer intake markers were divided into four groups, originating from (1) hops, present only in hops extract and test beers, (2) wort, present only in beer wort and test beers, (3) fermentation related markers, present only in beer but not in hops or wort, (4) human metabolism of beer components, present only in urine and plasma but not in beers, wort or hops. Since beer is a processed and complex beverage, none of the markers originating from beer raw materials or their processing individually may suffice as sufficiently specific and sensitive beer intake biomarkers. An aggregated biomarker combining each raw material and process was therefore constructed based on the principle that each included biomarker must be identified chemically and present as a signalling exposure marker clearly increasing after intake of each kind of beer while having much smaller or undetectable levels in the control period.

The calibration set for this aggregated biomarker was built from MSt1 including only the measurements of the four selected metabolite groups in the $24 \mathrm{~h}$ pooled urines from each period and 
from each of the 18 subjects. A PLSDA model with one component was established as the calibration model. The performance of the calibration model was evaluated using metabolome profiles from MSt2 (validation set), which had not been used in model development and optimization. The validation set included the measurements of the four selected metabolite groups in urine samples collected before (as control) vs. 0-2 h, 2-3 h and 3-12 h after beer intake (representing beer intake) for all four individuals.

\section{Results}

Recruitment started April 2013 and the trials ended November 2013. A total of 18 completers in MSt1 were included in the analyses. One subject dropped out after the first out of four periods, because she did not have enough time to participate anymore. All four completers in MSt2 were included. Data acquisition ended in August 2014. The trials ended as scheduled. We report here only the primary and some secondary aims according to the protocol. Baseline characteristics of the subjects are recorded in Table 1. No adverse events for the trial were recorded.

Table 1. Participant characteristics of MSt1 and MSt2 at the time of enrollment

\begin{tabular}{lcc}
\hline & MSt1 (n= 19) & MSt2 (n=4) \\
\hline Female [ $\mathrm{n}(\%)]$ & $9(47)$ & $2(50)$ \\
Age Range & $24-50$ & $28-60$ \\
BMI & $23.3 \pm 5.1$ & $22.4 \pm 3.4$ \\
Blood Pressure (mm Hg) & & \\
Systolic blood pressure & $110.2 \pm 6.5$ & $113.7 \pm 8.1$ \\
Diastolic blood pressure $_{\text {Habitual Alcohol Consumption }}^{1}[\mathrm{n}]$ & $62.0 \pm 8.0$ & $75.7 \pm 11.4$ \\
Moderate (1-2 drink/day) $^{\text {Low (or abstained) (<1 drink/day) }}$ & 11 & \\
\hline
\end{tabular}

${ }^{1}$ The levels of alcohol consumption is adapted from on NIH

Fasting whole-blood glucose and serum insulin levels were not different between treatments. There were no significant differences between treatments when glucose and insulin response were expressed in terms of total or incremental AUC. 


\section{Selection of metabolites associated with beer intake}

The number of samples for plasma was 216 (i.e. 18 subjects $\times$ three time points $\times$ four test drinks) and 360 for urine samples (i.e. 18 subjects $\times($ four time points + pool $) \times$ four test drinks). A total of 1503 and 8935 features remained after feature filtering for plasma and urine samples, respectively.

ASCA partitioned the total variation into contributions of corresponding experimental factors (i.e. test drink and time) and their interaction. A permutation test was applied in order to validate the ASCA factor models. Comparing the SS of 100,000 random class assignments with SS calculated from the original classes for each effect matrix, revealed that only the two main effects, time and test drink, were significant $(\mathrm{P}<0.0001)$, but not the interaction between time and test drink $(\mathrm{P}>0.7)$ (Figure $\mathrm{S}-1$ and $\mathrm{S}$ 2). The percentage of explained variation of each factor was calculated as the SS of decomposed factor matrices (Table S-1).

SCA scores plot (Figure 1) displays the separation of the samples at the test drink level both for urine and plasma. For urine, the separation of beer samples was mainly explained by PC1, with lower PC1 score values for beer samples compared to C. On the other hand, for plasma the dominating factor was alcohol, explained by PC1, and the combination of PC1 and PC2 provides the separation of beer intake.

SCA scores plots for individual factors and their interaction are also given in Supplemental Material (Figures S-3 and 4). 


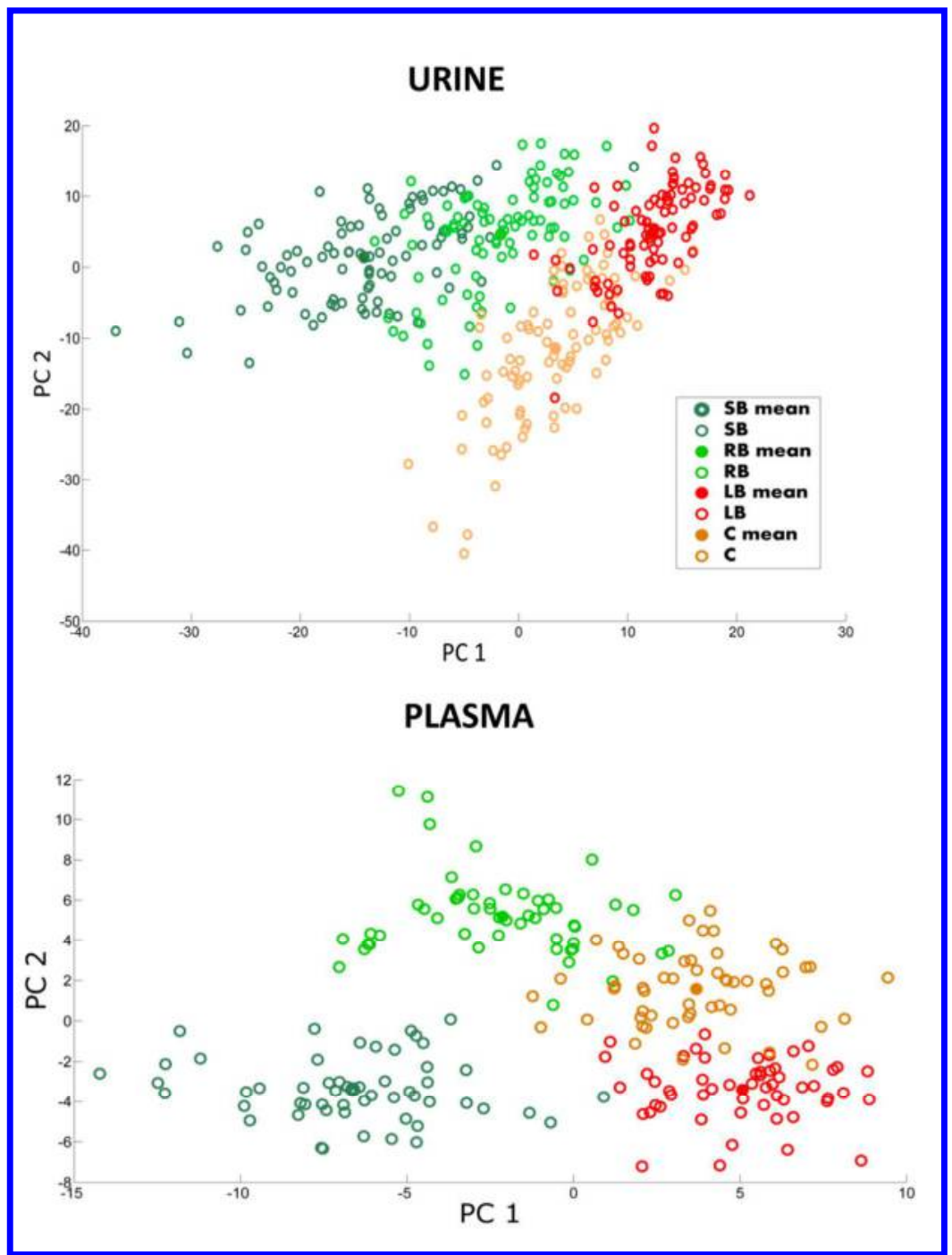

Figure 1. SCA score plot of the test drink matrix for both the urine (top) and plasma (bottom) metabolic profiles. An SCA model was applied to the effect matrix obtained from the ANOVA decomposition.

PLSDA was applied on the matrix where residuals were added to the decomposed test drink factor matrix. Three beers are assigned as one group providing a binary classification problem (i.e. $\mathrm{SB}+\mathrm{PB}+\mathrm{LB}$ vs. $\mathrm{C})$. The mean of misclassification errors of selected test sets for plasma and urine were significantly higher than classifications errors of 1000 random class assignments (P-values were 0.04 
for plasma and 0.004 for urine). The potential beer intake markers were selected by evaluating VIP values and selectivity ratio.

\section{Assessment of the origin of putative beer intake biomarkers}

Sixty nine compounds associated with beer intake were grouped based on their presence in test beers, wort and hops extracts as described in the Materials and Methods section. This comparison revealed 15 compounds originating directly from the hops extract (two in both plasma and urine, 13 only in urine), 19 directly from wort (one in both plasma and urine, three only in plasma, 14 only in urine), 2 from fermentation (only observed in urine) and 20 from human biotransformation (one only in plasma and 19 only in urine). The identified compounds are given in Table 2 and the list of unknown compounds and their source is given in Table S-2.

Table 2. Identified plasma and urine metabolites originating from beer raw materials hops and wort, fermentation process and human and/or microbial biotransformation of beer components.

\begin{tabular}{|c|c|c|c|c|c|c|c|}
\hline No & $\mathbf{m} / \mathbf{z}$ & RT & MS/MS & Annotation & $\begin{array}{l}\text { Suggested } \\
\text { Compound* }\end{array}$ & Biofluid & $\begin{array}{l}\text { Specificity to } \\
\text { beer intake/ } \\
\text { Source** }\end{array}$ \\
\hline 1 & 363.18 & $\begin{array}{l}4.08 \\
4.17 \\
4.2 \\
4.24\end{array}$ & $\begin{array}{l}\text { too low } \\
\text { signal }\end{array}$ & {$[\mathrm{M}-\mathrm{H}]^{-}$} & $\begin{array}{l}\text { Hydroxy alloiso- } \\
\text { cohumulone and/or } \\
\text { cohumulinone }^{3}\end{array}$ & Urine & S/hops \\
\hline 2 & 365.19 & $\begin{array}{l}4.15 \\
4.22 \\
4.27\end{array}$ & $\begin{array}{l}347.1,269.1 \\
251.1,237.1 \\
209.1,193.1 \\
181,141\end{array}$ & {$[\mathrm{M}-\mathrm{H}]^{-}$} & $\begin{array}{l}\text { Tetra-cyclocohumol } \\
\text { and/or tri- } \\
\text { cyclocohumol }^{3}\end{array}$ & Urine & S/hops \\
\hline 3 & $\begin{array}{l}377.182 \\
379.192\end{array}$ & $\begin{array}{l}4.27 \\
4.34 \\
4.2\end{array}$ & $\begin{array}{l}\text { too low } \\
\text { signal }\end{array}$ & $\begin{array}{l}{[\mathrm{M}-\mathrm{H}]^{-}} \\
{[\mathrm{M}+\mathrm{H}]^{+}}\end{array}$ & $\begin{array}{l}\text { Hydroxy alloiso- } \\
\text { ad/humulones } \\
\text { and/or } \\
\text { ad/humulinone }\end{array}$ & Urine & S/hops \\
\hline 4 & 379.21 & 4.29 & $\begin{array}{l}361.2,310.1 \\
283.1,265.1 \\
223.1,207.1\end{array}$ & {$[\mathrm{M}-\mathrm{H}]^{-}$} & $\begin{array}{l}\text { Tetra- } \\
\text { cycload/humol }\end{array}$ & Urine & S/hops \\
\hline
\end{tabular}




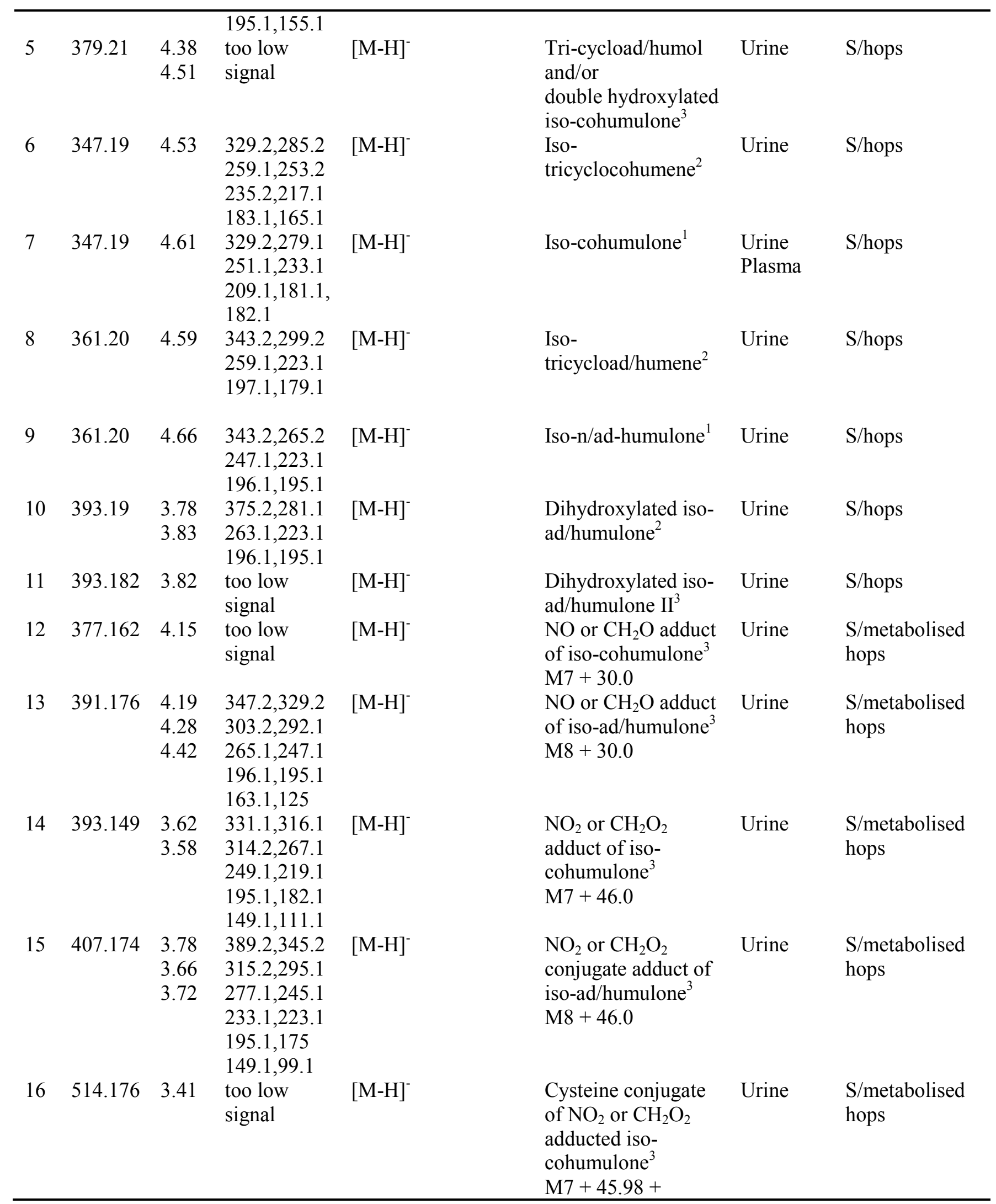




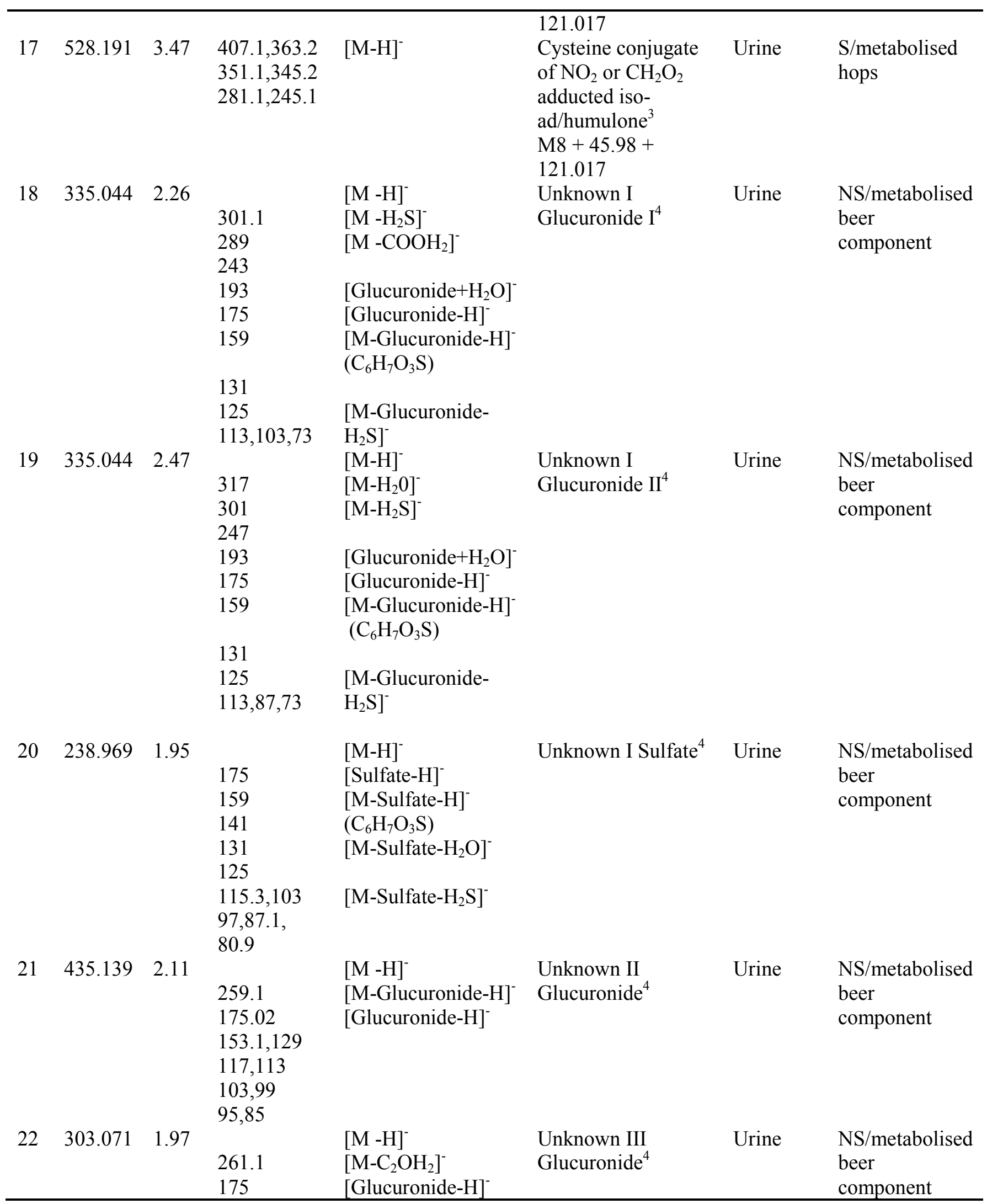




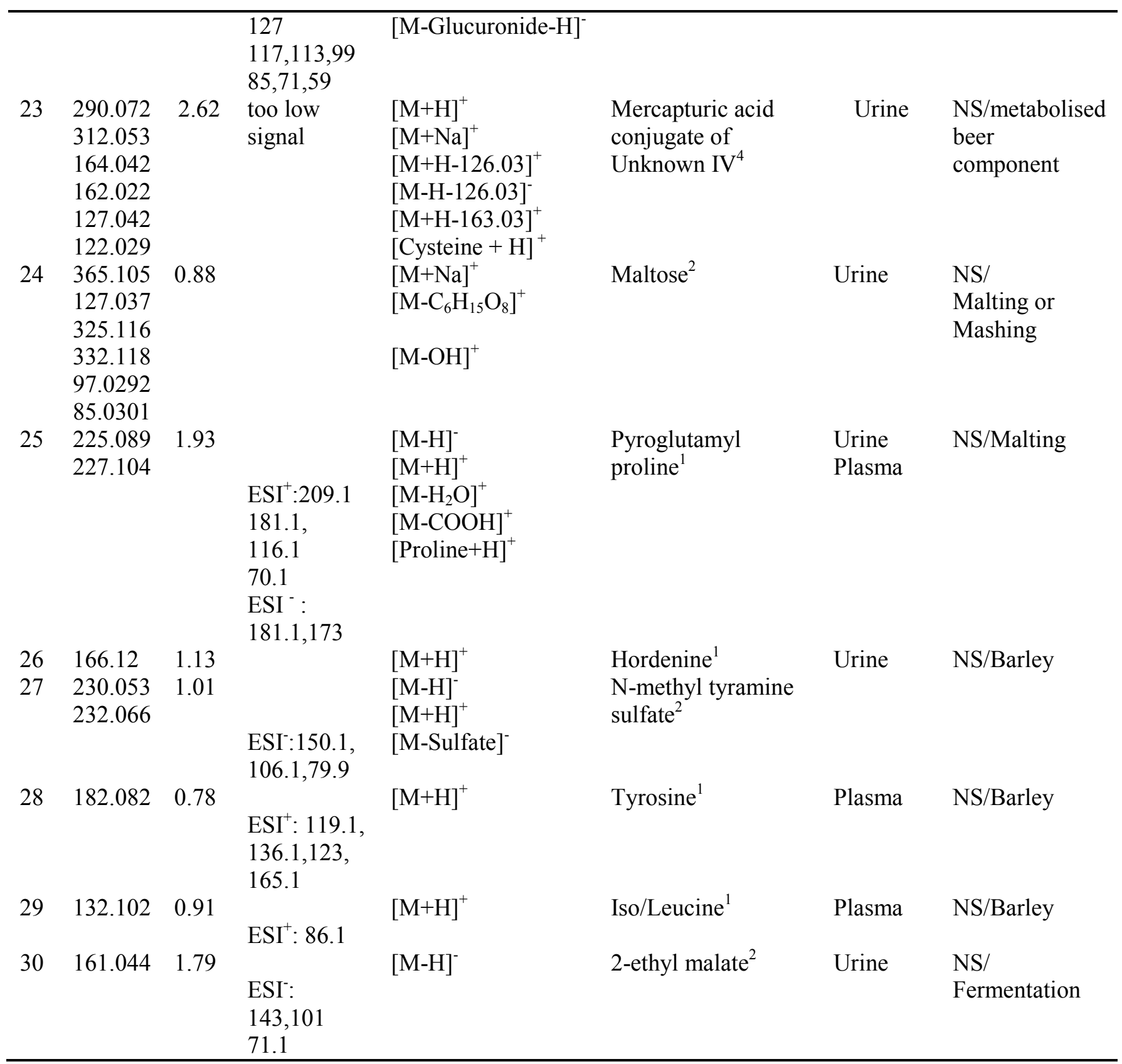

*identification level, ** source of unconjugated compound, S: Specific to beer intake; NS: Not specific to beer intake, UI: Unidentified

Identification of metabolites associated with beer intake. 
Metabolites originating from hops: The identified metabolites originating from hops were IAAs and their oxidation and degradation products (Table 2, Metabolites 1 to 11). Detailed description of identification based on MS/MS is provided in the Supplementary material (Text S-2, Figure S-5 and S6). IAAs, iso-cohumulone and the mixture of iso-humulone and iso-adhumulone were identified as markers of beer intake both in urine and plasma with very similar excretion profiles. Figure 2 illustrates the excretion profiles of iso-cohumulone after consumption of $330 \mathrm{~mL}$ of test drinks. In accordance to the measured IAA concentrations in test beers (Figure 2C), IAAs in urine and plasma were the highest following consumption of SB. In plasma they dropped rapidly within $3 \mathrm{~h}$, on the other hand, IAAs peaked later in urine (pooled urine within 1.5-3 h) and were still present in urine collected from $3 \mathrm{~h}$ up to $21 \mathrm{~h}$. 


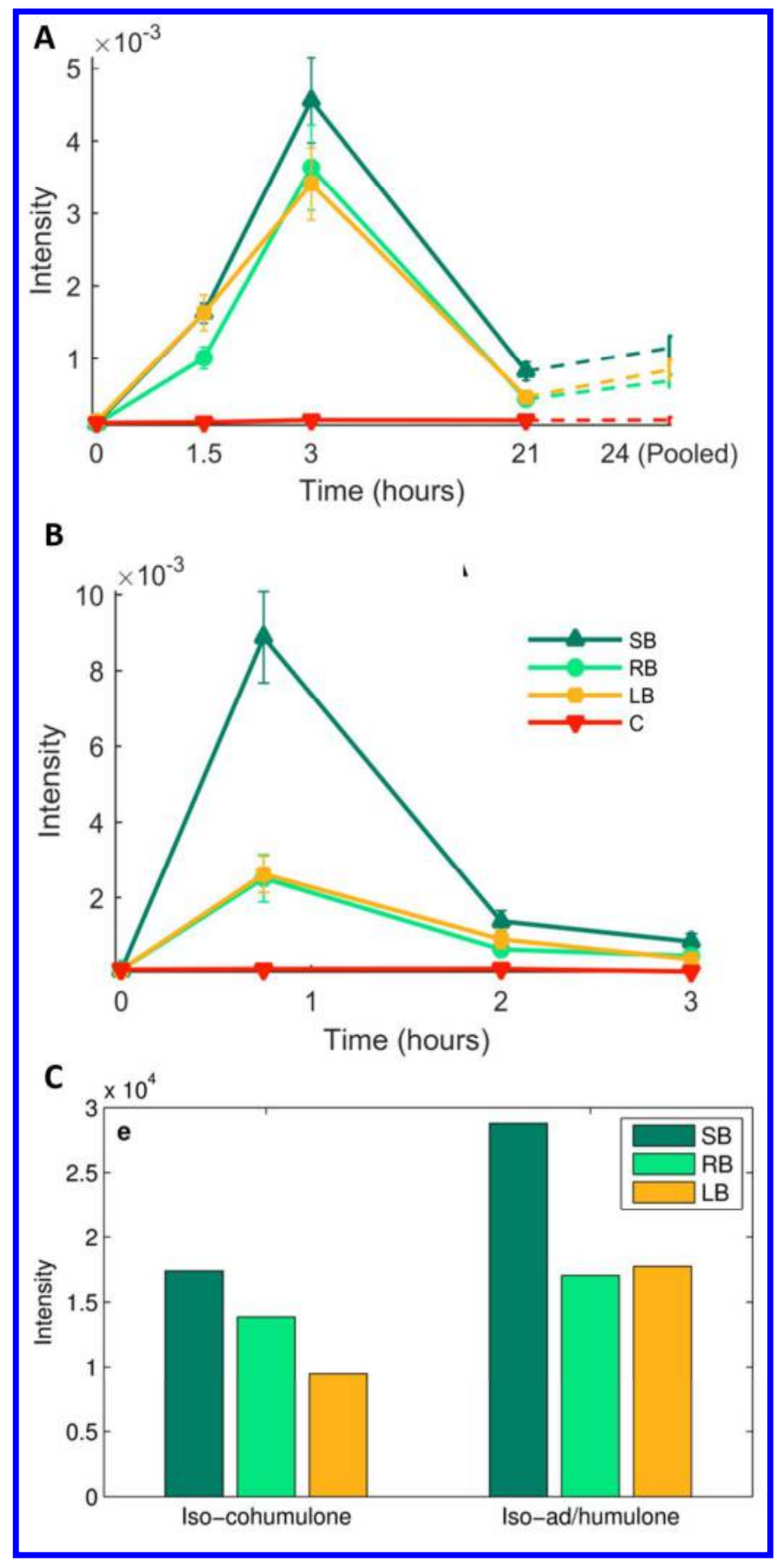

Figure 2. Iso-cohumulone excretion profiles in urine (A) plasma (B) and iso-cohumulone levels in test beers (C). 
Metabolites originating from human metabolism: Major metabolites were related to IAA biotransformation products and the same conjugates were recorded both for iso-cohumulone and isoad/humulones (Table 2, Metabolites 12 to 23). Three related groups of conjugates were observed with addition of $29.98 \mathrm{Da}$ (equal to addition of $\mathrm{NO}$ or $\mathrm{CH}_{2} \mathrm{O}$; metabolites 12 and 13), $45.98 \mathrm{Da}$ (equal to $\mathrm{NO}_{2}$ or $\mathrm{CH}_{2} \mathrm{O}_{2}$; metabolites14 and 15) and $166.99 \mathrm{Da}$ (metabolites 16 and 17) to iso-cohumulone and iso-ad/humulone. We speculate that these metabolites are oxidation and methylation products as well as cysteine adducts of isocohumulone and iso/adhumulone. Urinary excretion profiles of the IAA conjugates were similar to those of IAAs (Figure 2). Metabolites 15 and M16 were still present at high levels in urine collected between 3 and $21 \mathrm{~h}$, indicating that the peak of excretion of those metabolites was later than $3 \mathrm{~h}$ after intake (Figure S-8).

Metabolites 18 to 22 (Table 2) were products of phase II metabolism (glucuronides and sulfates) based on their fragmentation patterns, yet the identity of the biotransformed beer components remains unknown. Metabolites 20 to 22 were phase II (glucuronide and sulfate) metabolites of the same compound and their fragmentation pattern revealed the presence of a thiol group. Furthermore, one cysteine mercapturic acid conjugate (Metabolite 23) is detected as a urinary marker. In terms of excretion profiles, all of them peaked in urine collected within 1.5 to $3 \mathrm{~h}$. The products were not specific to the beer intake as they were also present after intake of $\mathrm{C}$, albeit at much lower levels (Figure S-8).

Metabolites originating from wort and fermentation: The identified metabolites are given in Table 2 (Metabolites 24 to 30). The only metabolite originating from wort that is detected in both urine and plasma was pGlu-Pro. NMR based structure elucidation of pGlu-Pro is described in Figure S-9. The urinary excretion profile is given in Figure 3A with the highest excretion level around $3 \mathrm{~h}$ following 
beer intake. The pGlu-pro was also detected in urine samples prior to the test meals and did not fluctuate following the consumption of $\mathrm{C}$ drink, indicating that it probably is a constituent of foods other than beer/wort or even that it is formed endogenously, but levels after beer intake are significantly higher. Hordenine, maltose (or an isomer) and NMT sulfate are identified as markers of beer intake in urine. Hordenine had already reached its maximum excretion level 0-1.5 h after intake, whereas maltose and NMT sulfate peaked in urine collected between 1.5-3 h and 3-21 h, respectively (Figure 3B, Figure S-10). Hordenine and NMT were present in the urine of the a few subjects prior to beer intake albeit with lower levels. 


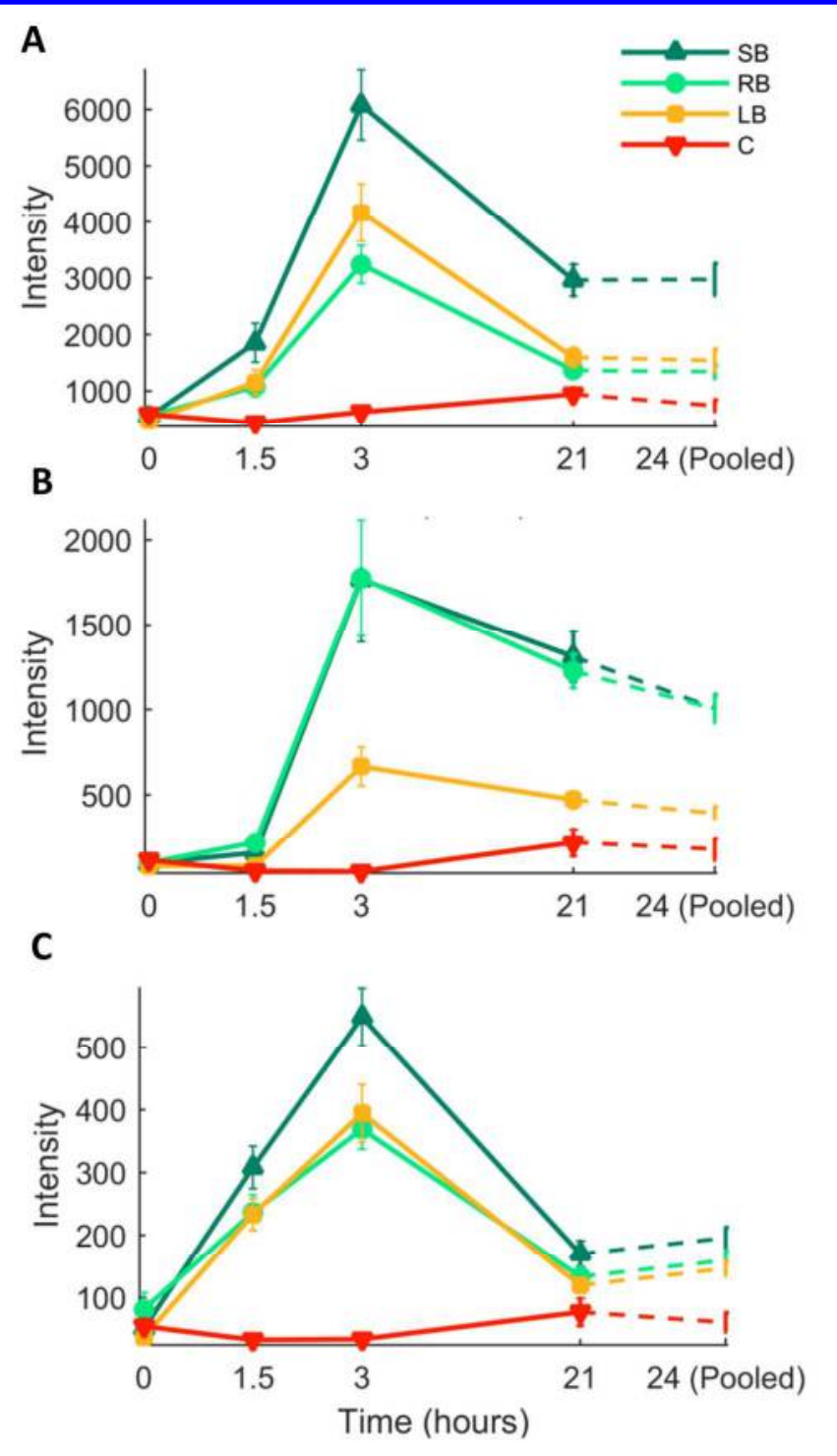

Figure 3. Urinary excretion profiles of pGlu-pro (A), NMT sulfate (B) and 2-ethyl malate (C).

In plasma, isoleucine, leucine and tyrosine are identified as metabolites originating from wort and associated with beer intake. The excretion profile of tyrosine is shown in Figure S-11, in agreement with tyrosine concentration in beer. Both tyrosine and isoleucine/leucine were observed as rapidly absorbed metabolites. 
In relation to fermentation, 2-ethyl malate is identified as a metabolite associated to beer intake in urine. It is excreted rather late compared to metabolites from hops and wort, with a reasonable high excretion between 3-21 h (Figure 3C).

\section{Selection of metabolites for the aggregated beer biomarker}

None of the beer intake markers discovered in this study was sufficiently specific or sensitive as single biomarkers to assess beer intake. The major issue with metabolites originating from hops (e.g. IAAs) is not specificity (see baseline levels, Figure 2) but sensitivity because they are not stable and several degradation products were also identified in urine at varying amounts. The issue with metabolites originating from wort or fermentation was that they were observed as markers of effect rather than exposure in the sense that they were present already at baseline but increased following beer intake. Hence none of the markers identified are specific to beer intake per se (see baseline levels, Figure 3). For the aggregated beer intake biomarker we selected one metabolite from each raw material (hops and barley) and production process (malting and fermentation) and established a compliance biomarker model for detection of beer intake based on MSt1. To overcome the issue with variable degradation of IAAs as representative of hops derived metabolites we used the sum of iso-cohumulone and isoad/humulones and their major degradation products, tricyclocohumol and tricyclohumol. NMT sulfate was selected representing barley and pGlu-pro to represent heat treatment during malting. Fermentation was represented by 2-ethyl malate since this compound is not removed in de-alcoholization processes to produce light and non-alcoholic beers.

Figure 4 demonstrates how the calibration model performs in terms of distinguishing samples after beer intake from the control time points and/or periods for calibration and validation sets. The scores plot of 
the first component (Figure 4A) demonstrates that the calibration model provides correct prediction of the classes as control and beer groups for the samples from MSt2 (open circles in Figure 4A) for urine collected up to $12 \mathrm{~h}$. Testing the same calibration model on urine samples collected from 12 to $24 \mathrm{~h}$ (MSt2), resulted in 0 sensitivity such that all the samples collected more than 12 hours after beer intake were wrongly classified as control samples (Figure S-12). This suggests that metabolites associated with beer intake were already excreted at $12 \mathrm{~h}$. Therefore, the calibration model is valid for detection of beer intake up to $12 \mathrm{~h}$ post consumption as well as in $24 \mathrm{~h}$ pooled urine samples.
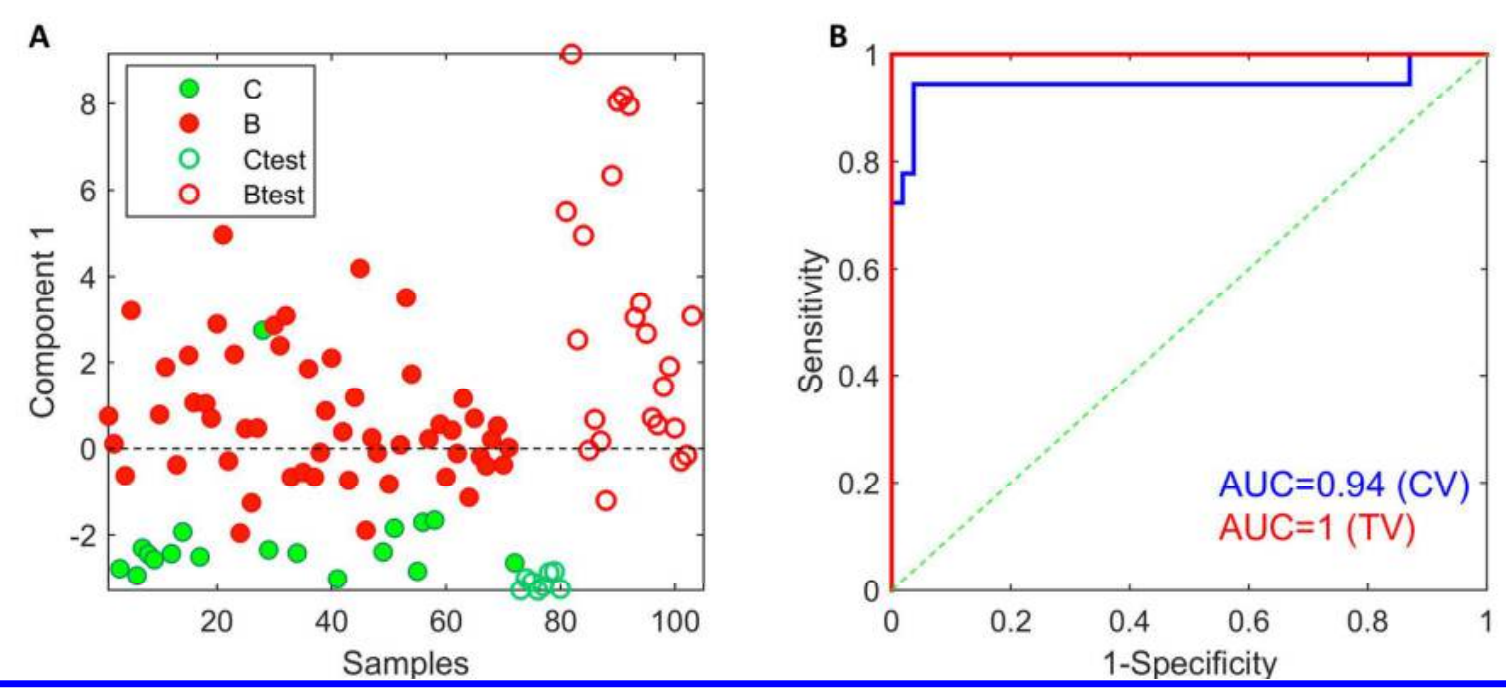

Figure 4 (A) PLSDA scores plot of the first component. The calibration model is developed using the measurements of four metabolites for samples from the meal study 1 (filled circles) and the model is tested with samples from meal study 2 (open circles). C and B refer to control samples and $24 \mathrm{~h}$ pooled samples from the meal the meal study 1, respectively. Ctest and Btest refer to the samples before and collected up to $12 \mathrm{~h}$ following beer intake from meal study 2 (B) ROC curve describing the performance of the PLSDA model in terms of predicting beer intake for calibration (CV) and test set (TV) samples, respectively. 
In order to assess the importance of each metabolite, we removed one of the four metabolites at a time and the calibration model still performed efficiently in terms of separating beer drinkers from control, thus none of the metabolites is individually crucial for the prediction.

\section{Discussion}

Accurate assessment of food intake is central in nutrition research and identification of specific food intake biomarkers is therefore a promising way ahead. Finding single biomarkers for complex foods like beer is difficult because each component in the food may be used also in other foods. The strategy described in the current study is therefore to derive aggregated biomarkers for complex foods adding the best markers for each component and processing step to improve the resulting biomarker specificity and sensitivity. Our cross-over meal study provided many markers associated with recent beer intake, particularly in urine, reflecting the metabolomes of beer, as well as metabolites originating from their human metabolism (Table 2). Beer metabolome is characterized by compounds originating from its raw materials, barley and hops, and their transformation products derived upon malting and fermentation. Each of the raw materials and processes flag specific compounds in urine and plasma. However, these are either present in other foods or unstable. Therefore individually they lack the specificity and sensitivity needed for intake biomarkers.

IAAs from hops have been previously suggested as biomarkers of beer intake as they are specific to beer $^{11,12,31}$. Although IAAs provided high sensitivity and specificity for detection of beer intake up to $21 \mathrm{~h}$ for MSt1, they were not even detected after intake of low hops beer (MSt2). This is attributed not only to the low hops content of the test beer but also to formation of several oxidative degradation products of IAAs ${ }^{32,33}$ underlining their instability. Tentatively identified degradation products of transIAA in urine were tri- and tetra-cyclohumenes and tri-cyclohumols ${ }^{14,34}$ whereas, mono- and di- 
hydroxylated humulones and humulinones appeared as oxygen-dependent degradation products of both cis- and trans-IAAs ${ }^{33}$. Indeed, only tricyclohumols were detected in low hops beer (MSt2), which has also been shown previously to be one of the major degradation products ${ }^{34}$. Therefore, using IAAs as single biomarkers of beer intake is not feasible since consumption of longer-term stored beers and beer varieties with low hops content may result in total degradation and therefore to low sensitivity for assessment of beer intake. Instead, the sum of IAAs and their major degradation products (e.i. tricyclohumols) as a single biomarker provides a better predictor of beer intake as potential loss of IAAs is partially compensated by formation of tri-cyclohumols. Although validating this biomarker in MSt2 provided high sensitivity and specificity $(\mathrm{AUC}=1)$, further studies including diverse types of beer and different storage periods are required for final validation. Our results also suggest that after ingestion IAAs are oxidized into metabolites, which could be tentatively identified based on fragmentation patterns (Table 2). The oxidized IAA metabolites are compounds similar to products of degradation during storage $\mathrm{e}^{35}$. It is difficult to discriminate between phase I metabolism and storage-induced oxidation of IAA as the products have very similar structural characteristics but IAA oxidation is much accelerated after ingestion indicating enzyme-mediated formation. Oral administration of IAAs to rabbits lead to recovery of less than $6 \%$ of the dose as such metabolites, suggesting that IAAs may also be subject to other kinds of metabolism ${ }^{16}$. Indeed, we observed unidentified conjugation products of IAAs formed from oxygenated IAAs by methylation and cysteine conjugation. Further isolation and structure elucidation is required to verify these structures. Some beer brands use hydrogenated hops extracts, which renders the IAAs more stable, however it is uncertain whether these are used exclusively since their organoleptic properties do not exactly match those of the normal hops extract ${ }^{36}$. Adding markers for these compounds might be needed to cover some more unusual brands of beer. 
The wort-derived beer intake markers were present in barley or formed during the malting/boiling process; therefore they may also be found in other barley containing foods, limiting their specificity. pGlu-pro was increased after beer intake, potentially due to a high content of proline in barley. pGlupro may be formed upon heating of the barley sprouts during malting or wort boiling. Pyroglutamyl peptides have been suggested to act on inflammatory diseases ${ }^{16,37,38}$ and have been shown to exhibit an antidepressant-like effect in mice ${ }^{39}$. To our knowledge, pGlu-Pro has not previously been observed in beer samples or associated with an increase in blood or urine after beer intake. The sulfate conjugate of NMT is another wort-derived compound, potentially formed through phase II metabolism in the plant or during the malting process. NMT is produced by N-methylation of tyramine, which is further methylated to hordenine ${ }^{40}$. Hordenine is suggested as a qualitative marker of beer intake ${ }^{41}$, yet its excretion profile after intake of other barley containing foods should be investigated since it may not be possible to discriminate beer intake from intake of other barley containing diets.

Malic acid, particularly in the form of di-ethyl malate is widely described as a constituent of alcoholic beverages $^{42,43}$, yet according to our knowledge, 2-ethyl malate has not previously been described in beer. We could also confirm its presence in wine and cider samples (data not shown), indicating that it is not specific to beer but to fermented beverages. 2-ethyl malate is probably formed through esterification of malic acid with ethanol, followed by partial rearrangement ${ }^{44}$ indicating that nonalcoholic food fermentation is probably not a source of its formation.

NMT sulfate, pGlu-pro and 2-ethyl malate were each detected in the urine of some subjects prior to intake of the test drink, suggesting intake from other food sources. Therefore, none of the single metabolites were sufficiently sensitive and specific for assessment of beer intake. Yet an aggregated biomarker characterizing the beer metabolome (i.e. its raw materials and the malting and brewing processes) provided a unique pattern. The four metabolites - NMT sulfate originating from barley, 2- 

ethyl malate originating from fermentation, pGlu-Pro originating from malting, and the sum of IAAs and their major degradation products (tricyclo-co- and -ad-humols) originating from hops - were used to establish an aggregated biomarker. It is crucial to validate new biomarkers with independent controlled trials as well as samples from observational studies ${ }^{45}$. Therefore, we utilized MSt2 for validation of the calibration model. The calibration model efficiently predicted beer intake up to $12 \mathrm{~h}$ $(\mathrm{AUC}=1$, Figure $4 \mathrm{~B})$; this set of four metabolites therefore provides an excellent aggregated biomarker for detection of beer intake. When we removed any one of the four metabolites from the aggregated biomarker, the calibration models performed equally well, suggesting that none of the individual metabolites are crucial for the model and that the aggregated approach is very robust.

Other compounds originating from hops, such as reduced derivatives of IAAs and prenylated phenols have been suggested previously as urinary and blood biomarkers of beer intake ${ }^{13,31,46}$. Isoxanthohumol was shown to be stable even after 10 years of storage, making it a suitable biomarker candidate. In our study, isoxanthohumol was only identified in hops extracts and beer samples (level 1), but not in urine and plasma (data not shown). This may be related to the low concentration of isoxanthohumol in urine and plasma; prenylated hops phenol glucuronide conjugates accounted for only $2 \%$ of the dose ${ }^{47,48}$. The gut microbial biotransformation of xanthohumol to prenylnaringenin in humans has been shown to vary from $0-100 \%$ between individuals ${ }^{49}$. This may also explain the relatively low sensitivity observed previously for this marker ${ }^{46}$.

\section{Conclusions}

The concept of aggregated biomarkers proposed here may find widespread use to solve the problems of overlapping markers for intakes of many foods and especially for processed foods and whole diets. A recent study demonstrated that aggregated biomarkers provide higher sensitivity and specificity for intake of sugar sweetened beverages compared to single biomarkers ${ }^{50}$. Further development of this 
concept will be to move it from the stage of providing qualitative markers to actually estimating the amount ingested and the time elapsed since ingestion. This should eventually be possible based on the different kinetics of the individual metabolites included in the aggregated biomarkers.

In conclusion, we were able to segregate metabolites that are increased following beer intake into compounds originating from hops, wort, fermentation, and human metabolism. The majority of beerspecific compounds originate from hops, but many of these are chemically unstable limiting their usefulness as single biomarkers. Many of the suggested biotransformation products of IAAs are novel, yet still need to be fully structurally characterized. We propose that wort and fermentation products may provide signature markers of beer intake in combination with one or more hops-specific marker. Therefore, we have shown here that an aggregated biomarker model based on a set of three out of four of these signature biomarkers was able to completely discriminate between samples collected after beer intake in an independent study with high sensitivity and specificity. It still remains to be shown that the aggregated biomarker of beer intake proposed herein is sensitive and specific in an observational setting and whether adjustments may be needed, e.g. adding markers for optional beer components such as reduced IAAs, caramel, wheat, corn, etc.

\section{Author Contributions}

GG, MGJ, EL and LOD designed the research; LOD conducted the study; MGJ, SM, LB and EL provided essential materials and advice; GG and MGJ analyzed the data; GG, SM, LOD interpreted the results GG and LOD wrote the manuscript.

\section{Notes}

The authors declare no competing financial interest. 


\section{Acknowledgements}

This study was supported by a grant from Carlsberg A/S and Foodball (BIO-NH call under the Joint Programming Initiative). FoodBAll is a project funded by the BIO-NH call under the Joint Programming Initiative, "a Healthy Diet for a Healthy Life". The project is funded nationally by the Danish Innovation Foundation (\#4203-00002B). None of the funding sources played a decisive role in the design, collection, analysis, or interpretation of the data or in the decision to submit the manuscript for publication. We thank Suzanne Runstedt, (Department of Nutrition, Exercise and Sports, University of Copenhagen), for writing the ethical protocol and managing the study; Ümmühan Celik (Department of Nutrition, Exercise and Sports, University of Copenhagen) for blood sampling and laboratory analyses; and Sarah Ben Soltane (Department of Nutrition, Exercise and Sports, University of Copenhagen) for laboratory analyses. All participants are thanked for their participation in the study. 


\section{Supporting Information}

Figure S-1. SSQ distribution calculated by permuting the class assignments and the SSQ calculated for original class assignments for time and test drink effects.

Figure S-2. SSQ distribution calculated by permuting the class assignments and the SSQ calculated for original class assignments for time-test drink interaction.

Text S-1. Identification of significant factors in the model

Figure S-3. ASCA decomposition of urine LC-MS profiles. SCA scores plots for (a) test drink, (b) time and (c) interaction of test drink and time for PC1.

Figure S-4. ASCA decomposition of plasma LC-MS profiles. SCA scores plots for (a) test drink, (b) time and (c) interaction of test drink and time for PC1.

Table S-1. Unknown metabolites associated with consumption of test beers.

Table S-2. Unknown metabolites associated with consumption of test beers.

Text S-2. Identification of hops-derived metabolites

Figure S-5. Extracted ion chromatogram for iso-cohumulone in ICS-I3, hops extract stored for 4 months, fresh hops extract, test beer (SB) and urine sample 3 hours after consumption of the test beer (SB)

Figure S-6. LC-MS/MS spectrum at 20V (ESI-) of iso-cohumulone and iso-/tricyclocohumene.

Figure S-7. Urinary excretion profiles of metabolites originating from hops.

Figure S-8. Urinary excretion profiles of unknown metabolites originating from human biotransformation observed after beer intake.

Figure S-9. NMR identification of pyroglutamyl-proline with ${ }^{1} \mathrm{H}$ and ${ }^{13} \mathrm{C}$ chemical shifts for the aliphatic $\mathrm{CH}$ groups in UPLC fraction containing several molecular species. . 
Figure S-10. Urinary excretion profiles of maltose originating from wort

Figure S-11. Tyrosine excretion profiles in plasma (a) and their corresponding levels in the test beers (b).

Figure S-12. PLSDA model developed including pooled $24 \mathrm{~h}$ samples from MSt1 and the performance of the model in terms of detection of beer intake between 12 and $24 \mathrm{~h}$ for MSt2. 


\section{References}

1. Koppes, L. L.; Dekker, J. M.; Hendriks, H. F.; Bouter, L. M.; Heine, R. J. Moderate alcohol consumption lowers the risk of type 2 diabetes: a meta-analysis of prospective observational studiesDiabetes Care 2005, 3, 719-725.

2. Ronksley, P. E.; Brien, S. E.; Turner, B. J.; Mukamal, K. J.; Ghali, W. A. Association of alcohol consumption with selected cardiovascular disease outcomes: a systematic review and meta-analysis. BMJ 2011, d671.

3. Bagnardi, V.; Rota, M.; Botteri, E.; Tramacere, I.; Islami, F.; Fedirko, V.; Scotti, L.; Jenab, M.; Turati, F.; Pasquali, E.; Pelucchi, C.; Bellocco, R.; Negri, E.; Corrao, G.; Rehm, J.; Boffetta, P.; La Vecchia, C. Light alcohol drinking and cancer: a meta-analysisAnn. Oncol. 2013, 2, 301-308.

4. Rimm, E. B.; Klatsky, A.; Grobbee, D.; Stampfer, M. J. Review of moderate alcohol consumption and reduced risk of coronary heart disease: is the effect due to beer, wine, or spirits $B M J 1996,7033$, 731-736.

5. Brien, S. E.; Ronksley, P. E.; Turner, B. J.; Mukamal, K. J.; Ghali, W. A. Effect of alcohol consumption on biological markers associated with risk of coronary heart disease: systematic review and meta-analysis of interventional studies. BMJ 2011, d636.

6. Zamora-Ros, R.; Urpí-Sardà, M.; Lamuela-Raventós, R. M.; Estruch, R.; Martínez-González, M. Á; Bulló, M.; Arós, F.; Cherubini, A.; Andres-Lacueva, C. Resveratrol metabolites in urine as a biomarker 
of wine intake in free-living subjects: The PREDIMED Study. Free Radical Biology and Medicine 2009, 12, 1562-1566.

7. Quifer-Rada, P.; Martinez-Huelamo, M.; Chiva-Blanch, G.; Jauregui, O.; Estruch, R.; LamuelaRaventos, R. M. Urinary Isoxanthohumol Is a Specific and Accurate Biomarker of Beer Consumption. J. Nutr. 2014, 4, 484-488.

8. Possemiers, S.; Bolca, S.; Grootaert, C.; Heyerick, A.; Decroos, K.; Dhooge, W.; De Keukeleire, D.; Rabot, S.; Verstraete, W.; Van De Wiele, T. The prenylflavonoid isoxanthohumol from hops (Humulus lupulus L.) is activated into the potent phytoestrogen 8-prenylnaringenin in vitro and in the human intestine. J. Nutr. 2006, 7, 1862-1867.

9. Nookandeh, A.; Frank, N.; Steiner, F.; Ellinger, R.; Schneider, B.; Gerhäuser, C.; Becker, H. Xanthohumol metabolites in faeces of rats. Phytochemistry 2004, 5, 561-570.

10. Cortacero-Ramırez, S.; de Castro, M. H.; Segura-Carretero, A.; Cruces-Blanco, C.; FernandezGutierrez, A. Analysis of beer components by capillary electrophoretic methods. TrAC Trends in Analytical Chemistry 2003, 7, 440-455.

11. Rodda, L. N.; Gerostamoulos, D.; Drummer, O. H. Pharmacokinetics of iso-alpha-acids in volunteers following the consumption of beer. J. Anal. Toxicol. 2014, 6, 354-359.

12. Rodda, L. N.; Gerostamoulos, D.; Drummer, O. H. The rapid identification and quantification of iso- $\alpha$-acids and reduced iso- $\alpha$-acids in blood using UHPLC-MS/MS: validation of a novel marker for beer consumption. Analytical and bioanalytical chemistry 2013, 30, 9755-9767. 
13. Intelmann, D.; Haseleu, G.; Dunkel, A.; Lagemann, A.; Stephan, A.; Hofmann, T. Comprehensive sensomics analysis of hop-derived bitter compounds during storage of beer. J. Agric. Food Chem. 2011, 5, 1939-1953.

14. Intelmann, D.; Kummerlöwe, G.; Haseleu, G.; Desmer, N.; Schulze, K.; Fröhlich, R.; Frank, O.; Luy, B.; Hofmann, T. Structures of Storage $\square$ Induced Transformation Products of the Beer's Bitter Principles, Revealed by Sophisticated NMR Spectroscopic and LC-MS Technique. Chemistry-A European Journal 2009, 47, 13047-13058.

15. Rodda, L. N.; Gerostamoulos, D.; Drummer, O. H. Pharmacokinetics of reduced iso-a-acids in volunteers following clear bottled beer consumption. Forensic Sci. Int. 2015, , 37-43.

16. Cattoor, K.; Dresel, M.; De Bock, L.; Boussery, K.; Van Bocxlaer, J.; Remon, J. -.; De Keukeleire, D.; Deforce, D.; Hofmann, T.; Heyerick, A. Metabolism of hop-derived bitter acids. J. Agric. Food Chem. 2013, 33, 7916-7924.

17. Koloverou, E.; Panagiotakos, D.; Pitsavos, C.; Chrysohoou, C.; Georgousopoulou, E.; Metaxa, V.; Stefanadis, C.; ATTICA Study Group Effects of alcohol consumption and the metabolic syndrome on 10-year incidence of diabetes: The ATTICA study. Diabetes Metab. 2015, 2, 152-159.

18. Janssens, J. P.; Shapira, N.; Debeuf, P.; Michiels, L.; Putman, R.; Bruckers, L.; Renard, D.; Molenberghs, G. Effects of soft drink and table beer consumption on insulin response in normal teenagers and carbohydrate drink in youngsters. Eur. J. Cancer Prev. 1999, 4, 289-295. 
19. Andersen, M. S.; Rinnan, Å; Manach, C.; Poulsen, S. K.; Pujos-Guillot, E.; Larsen, T. M.; Astrup, A.; Dragsted, L. O. Untargeted metabolomics as a screening tool for estimating compliance to a dietary pattern. Journal of proteome research 2014, 3, 1405-1418.

20. Rago, D.; Mette, K.; Gürdeniz, G.; Marini, F.; Poulsen, M.; Dragsted, L. O. A LC-MS metabolomics approach to investigate the effect of raw apple intake in the rat plasma metabolome. Metabolomics 2013, 6, 1202-1215.

21. Gürdeniz, G.; Rago, D.; Bendsen, N. T.; Savorani, F.; Astrup, A.; Dragsted, L. O. Effect of trans fatty acid intake on LC-MS and NMR plasma profiles. PloS one 2013, 7, e69589.

22. Scalbert, A.; Brennan, L.; Manach, C.; Andres-Lacueva, C.; Dragsted, L. O.; Draper, J.; Rappaport, S. M.; van der Hooft, J. J.; Wishart, D. S. The food metabolome: a window over dietary exposure. Am. J. Clin. Nutr. 2014, 6, 1286-1308.

23. Gürdeniz, G.; Kristensen, M.; Skov, T.; Dragsted, L. O. The effect of LC-MS data preprocessing methods on the selection of plasma biomarkers in fed vs. fasted rats. Metabolites 2012, 1, 77-99.

24. Andersen, M. B. S.; Reinbach, H. C.; Rinnan, A.; Barri, T.; Mithril, C.; Dragsted, L. O. Discovery of exposure markers in urine for Brassica-containing meals served with different protein sources by UPLC-qTOF-MS untargeted metabolomics. Metabolomics 2013, 5, 984-997.

25. Barri, T.; Holmer-Jensen, J.; Hermansen, K.; Dragsted, L. O. Metabolic fingerprinting of high-fat plasma samples processed by centrifugation-and filtration-based protein precipitation delineates significant differences in metabolite information coverage. Anal. Chim. Acta 2012, , 47-57. 
26. Smilde, A. K.; Jansen, J. J.; Hoefsloot, H. C.; Lamers, R. J.; van der Greef, J.; Timmerman, M. E. ANOVA-simultaneous component analysis (ASCA): a new tool for analyzing designed metabolomics data. Bioinformatics 2005, 13, 3043-3048.

27. Vis, D. J.; Westerhuis, J. A.; Smilde, A. K.; van der Greef, J. Statistical validation of megavariate effects in ASCA. BMC Bioinformatics 2007, 322.

28. Zwanenburg, G.; Hoefsloot, H. C.; Westerhuis, J. A.; Jansen, J. J.; Smilde, A. K. ANOVAprincipal component analysis and ANOVA-simultaneous component analysis: A comparison. $J$. Chemometrics 2011, 10, 561-567.

29. Szymańska, E.; Saccenti, E.; Smilde, A. K.; Westerhuis, J. A. Double-check: validation of diagnostic statistics for PLS-DA models in metabolomics studies. Metabolomics 2012, 1, 3-16.

30. Sumner, L. W.; Amberg, A.; Barrett, D.; Beale, M. H.; Beger, R.; Daykin, C. A.; Fan, T. W.; Fiehn, O.; Goodacre, R.; Griffin, J. L. Proposed minimum reporting standards for chemical analysis.

Metabolomics 2007, 3, 211-221.

31. Rodda, L. N.; Gerostamoulos, D.; Drummer, O. H. Pharmacokinetics of reduced iso- $\alpha$-acids in volunteers following clear bottled beer consumption. Eorensic Sci.Int. 2015, 37-43.

32. Cooman, L.; Aerts, G.; Overmeire, H.; Keukeleire, D. Alterations of the Profiles of Iso- $\alpha$-Acids During Beer Ageing, Marked Instability of Trans-Iso- $\alpha$-Acids and Implications for Beer Bitterness Consistency in Relation to Tetrahydroiso- $\alpha$-Acids. J. Inst. Brewing 2000, 3, 169-178. 
33. Intelmann, D.; Hofmann, T. On the autoxidation of bitter-tasting iso- $\alpha$-acids in beer. J. Agric. Food Chem. 2010, 8, 5059-5067.

34. Schmidt, C.; Biendl, M.; Lagemann, A.; Stettner, G.; Vogt, C.; Dunkel, A.; Hofmann, T. Influence of Different Hop Products on the cis/trans Ratio of Iso- $\alpha$-Acids in Beer and Changes in Key Aroma and Bitter Taste Molecules During Beer Ageing. J.Am.Soc.Brew.Chem 2014, 2, 116-125.

35. Cattoor, K.; Dresel, M.; De Bock, L.; Boussery, K.; Van Bocxlaer, J.; Remon, J.; De Keukeleire, D.; Deforce, D.; Hofmann, T.; Heyerick, A. Metabolism of Hop-Derived Bitter Acids. J. Agric. Food Chem. 2013, 33, 7916-7924.

36. Weiss, A.; Schönberger, C.; Mitter, W.; Biendl, M.; Back, W.; Krottenthaler, M. Sensory and analytical characterisation of reduced, isomerised hop extracts and their influence and use in beer. $J$. Inst. Brewing 2002, 2, 236-242.

37. Hirai, S.; Horii, S.; Matsuzaki, Y.; Ono, S.; Shimmura, Y.; Sato, K.; Egashira, Y. Antiinflammatory effect of pyroglutamyl-leucine on lipopolysaccharide-stimulated RAW 264.7 macrophages. Life Sci. 2014, 1, 1-6.

38. Sato, K.; Egashira, Y.; Ono, S.; Mochizuki, S.; Shimmura, Y.; Suzuki, Y.; Nagata, M.; Hashimoto, K.; Kiyono, T.; Park, E. Y. Identification of a hepatoprotective peptide in wheat gluten hydrolysate against D-galactosamine-induced acute hepatitis in rats. J. Agric. Food Chem. 2013, 26, 6304-6310.

39. Yamamoto, Y.; Mizushige, T.; Mori, Y.; Shimmura, Y.; Fukutomi, R.; Kanamoto, R.; Ohinata, K. Antidepressant-like effect of food-derived pyroglutamyl peptides in mice. Neuropeptides 2015, ,25-29. 
40. Lovett, J. V.; Hoult, A. H.; Christen, O. Biologically active secondary metabolites of barley. IV. Hordenine production by different barley lines. J. Chem. Ecol. 1994, 8, 1945-1954.

41. Steiner, I.; Brauers, G.; Temme, O.; Daldrup, T. A sensitive method for the determination of hordenine in human serum by ESI UPLC-MS/MS for forensic toxicological applications. Analytical and bioanalytical chemistry 2016, 9, 2285-2292.

42. Ivanova, V.; Stefova, M.; Vojnoski, B.; Stafilov, T.; Bíró, I.; Bufa, A.; Felinger, A.; Kilár, F. Volatile composition of Macedonian and Hungarian wines assessed by GC/MS. Food and Bioprocess Technology 2013, 6, 1609-1617.

43. Webb, A. D.; Kepner, R. E.; Maggiora, L. Identification of ethyl acid tartrate and one isomer of ethyl acid malate in California flor sherryJ. Agric. Food Chem. 1967, 2, 334-339.

44. Amer, M.; Novoa-Díaz, D.; Puig-Pujol, A.; Capdevila, J.; Chávez, J.; Turó, A.; García-Hernández, M.; Salazar, J. Ultrasonic velocity of wáter-ethanol-malic acid-lactic acid mixtures during the malolactic fermentation process. J. Food Eng. 2015, , 61-69.

45. Kuhnle, G. G. Nutritional biomarkers for objective dietary assessment. J. Sci. Food Agric. 2012, 6, 1145-1149.

46. Quifer-Rada, P.; Martinez-Huelamo, M.; Chiva-Blanch, G.; Jauregui, O.; Estruch, R.; LamuelaRaventos, R. M. Urinary isoxanthohumol is a specific and accurate biomarker of beer consumption. $J$. Nutr. 2014, 4, 484-488. 
47. Legette, L.; Karnpracha, C.; Reed, R. L.; Choi, J.; Bobe, G.; Christensen, J. M.; Rodriguez-Proteau, R.; Purnell, J. Q.; Stevens, J. F. Human pharmacokinetics of xanthohumol, an antihyperglycemic flavonoid from hops. Molecular nutrition \& food research 2014, 2, 248-255.

48. van Breemen, R. B.; Yuan, Y.; Banuvar, S.; Shulman, L. P.; Qiu, X.; Alvarenga, R. F. R.; Chen, S.; Dietz, B. M.; Bolton, J. L.; Pauli, G. F. Pharmacokinetics of prenylated hop phenols in women following oral administration of a standardized extract of hops. Molecular nutrition \& food research 2014, 10, 1962-1969.

49. Possemiers, S.; Bolca, S.; Grootaert, C.; Heyerick, A.; Decroos, K.; Dhooge, W.; De Keukeleire, D.; Rabot, S.; Verstraete, W.; Van De Wiele, T. The prenylflavonoid isoxanthohumol from hops (Humulus lupulus L.) is activated into the potent phytoestrogen 8-prenylnaringenin in vitro and in the human intestine. J. Nutr. 2006, 7, 1862-1867.

50. Gibbons, H.; McNulty, B. A.; Nugent, A. P.; Walton, J.; Flynn, A.; Gibney, M. J.; Brennan, L. A metabolomics approach to the identification of biomarkers of sugar-sweetened beverage intake. Am.J. Clin. Nutr. 2015, 3, 471-477. 


\section{Figure Legends}

Figure 1. SCA score plot of the test drink matrix for both the urine (top) and plasma (bottom) metabolic profiles. An SCA model was applied to the effect matrix obtained from the ANOVA decomposition.

Figure 2. Iso-cohumulone excretion profiles in urine (A) plasma (B) and test beer levels (C).

Figure 3. Urinary excretion profiles of pGlu-pro (A), NMT sulfate (B) and 2-ethyl malate (C).

Figure 4 (A) PLSDA scores plot of the first component. The calibration model is developed using the measurements of four metabolites for samples from the meal study 1 (filled circles) and the model is tested with samples from meal study 2 (open circles). C and B refer to control samples and $24 \mathrm{~h}$ pooled samples from the meal the meal study 1, respectively. Ctest and Btest refer to the samples before and collected up to $12 \mathrm{~h}$ following beer intake from meal study 2 (B) ROC curve describing the performance of the PLSDA model in terms of predicting beer intake for calibration (CV) and test set (TV) samples, respectively. 


\section{Table Legends}

Table 1. Participant characteristics of MSt1 and MSt2 at the time of enrollment

Table 2. Identified plasma and urine metabolites originating from beer raw materials hops and wort, fermentation process and human and/or microbial biotransformation of beer components. 
The image [in TOC graphic] is free domain and permission is not required

\section{For Table Contents Only}

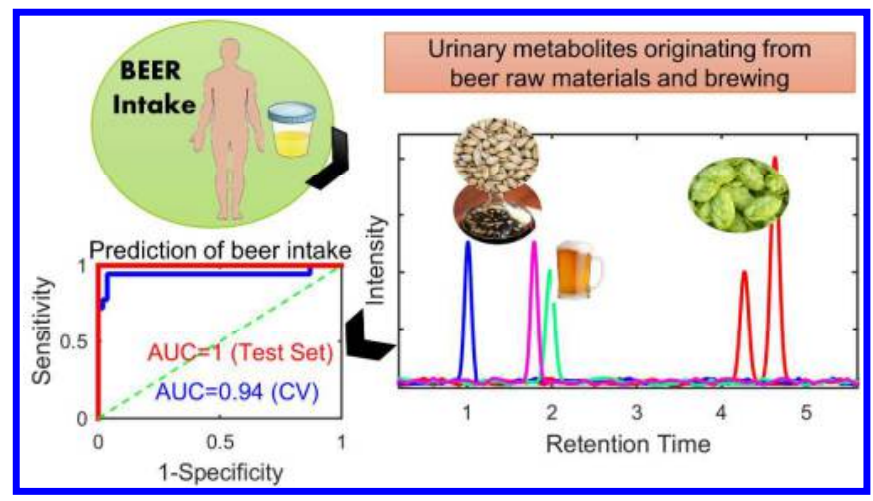

34

35

36

37

38

39

40

41

42

43

44

45

46

47

48

49

50

51

52

53

54

55

56

57

58

59

60 